\section{O impacto do trabalho em saúde mental: transtornos psiquiátricos menores, qualidade de vida e satisfação profissional}

\section{Job impact on mental health workers: minor psychiatric disorders, quality of life and job satisfaction}

Patrícia Furuta De Marco', Vanessa de Albuquerque Cítero', Edilaine Moraes', Luiz Antonio Nogueira-Martins'

\section{RESUMO}

Objetivo: Avaliar o impacto da carga de trabalho sobre a satisfação profissional, a qualidade de vida e a prevalência de transtornos psiquiátricos menores em profissionais de saúde mental. Método: Estudo transversal com amostra de 203 profissionais de saúde mental. Foram aplicados os instrumentos: IMPACTO-BR, SATIS-BR, SRQ-20 e um questionário sociodemográfico ocupacional. Resultados: 75,4\% dos participantes eram do sexo feminino, com idade média de 33,7 anos (DP $=9) ; 15,8 \%$ dos participantes apresentaram transtornos psiquiátricos menores. Em relação ao trabalho, obteve-se nível de satisfação global mediano (escore 3,59; DP = 0,485). O impacto global foi avaliado como pequeno (escore 1,85; DP = 0,536). Sobre a qualidade de vida, houve prejuízo nos subitens dor, estado geral de saúde e vitalidade. Conclusão: Os dados obtidos com esta pesquisa demonstram que os profissionais que atuam na área da saúde mental sentem-se mais satisfeitos em relação à qualidade dos serviços oferecidos e ao trabalho realizado em equipe. O fator que gerou menor satisfação está relacionado às condições de trabalho oferecidas. Foram observados escores mais elevados na subescala referente ao impacto emocional, assim como maior presença de transtornos psiquiátricos menores nos profissionais de terapia ocupacional e serviço social. Profissionais que trabalham mais de 20 horas semanais apresentam prejuízos na qualidade de vida..

\begin{abstract}
Objective: To evaluate the impact of workload over professional satisfaction, quality of life and minor psychiatric disorders among mental health professionals. Method: 203 mental health professionals were enrolled in a cross-sectional study. The following instruments have been used: IMPACTO-BR; SATIS-BR; SRQ-20; SF-36; and a socio-demographic occupational questionnaire. Results: $75.4 \%$ of the participants were female; the mean age was 33.7 years $(S D=9) ; 15.8 \%$ had minor psychiatric disorders. The global satisfaction score with the workload was considered medium (3.59; SD $=0.485)$ and the global work impact score was small $(1.85 ; S D=0.536)$. It was observed low score on quality of life in the subitens: pain, general health state and vitality. Conclusion: The study showed that professionals who work in the mental health area have been professionally satisfied with the quality of services offered to the patient and teamwork. The lowest satisfaction score was related to bad working conditions. The highest satisfaction scores were related to the emotional burden, and higher prevalence of minor psychiatric disorders of occupational therapists and social service professionals. Health professionals who worked more than 20 weekly hours showed worse quality of life.
\end{abstract}

1 Departamento de Psiquiatria da Universidade Federal de São Paulo (Unifesp).

Endereço para correspondência: Patrícia Furuta De Marco Rua Tucuna, 969, apto. 111, Vila Pompéia - 05021-010 - São Paulo, SP

E-mail: patfuruta@uol.com.br 
A ampla mudança do atendimento público em saúde mental, derivada da reforma da assistência psiquiátrica brasileira ocorrida efetivamente a partir das novas políticas de saúde instituídas pelo Ministério da Saúde, em 2001 (Lei Federal no 10.216, de 6 de abril de 2001), no Brasil', gerou demanda pela criação de novos serviços especializados em atenção comunitária e pública em saúde mental, como os centros de assistência psicossocial, serviços residenciais terapêuticos e unidades psiquiátricas em hospitais gerais, e em conseqüência deste crescimento, a busca por profissionais capacitados.

No entanto, a falta de investimentos em recursos humanos no setor da saúde mental vem agravando-se, gerando falta de profissionais qualificados, achatamento salarial e não-reposição de pessoal². Este fato, além de ocasionar contratações de temporários, cooperativas de profissionais, serviços terceirizados e, até mesmo, trabalho voluntário, contribui para o aumento da sobrecarga de trabalho sentida pela equipe, altos níveis de estresse, burnout e sintomas depressivos e ansiosos ${ }^{2-6}$

A Organização Mundial de Saúde (OMS) faz importantes recomendações no que concerne às mudanças relacionadas à reforma psiquiátrica?

Uma das preocupações está vinculada à qualidade dos serviços prestados que beneficie os pacientes de serviços de saúde mental e seus familiares, reduzindo progressivamente as internações e capacitando, aos poucos, os pacientes a se integrarem novamente à sociedade, ampliando as possibilidades destes ao se identificarem neste novo contexto.

Para cumprir estas diretrizes, as características e a forma de organização da tarefa assistencial têm sido constantemente debatidas. As políticas de saúde mental vigentes no Brasil recomendam que o atendimento dos pacientes psiquiátricos seja efetuado por equipes multidisciplinares, atuando na perspectiva de realização de diversas tarefas que incluem a busca de adesão ao tratamento, o controle da sintomatologia, a orientação familiar e comunitária, a articulação entre os serviços assistenciais e a avaliação de programas ${ }^{8}$.

Segundo Bandeira9', a avaliação dos serviços de saúde mental recebeu atenção crescente por causa do incentivo da OMS em aumentar a qualidade dos serviços de saúde mental. Nesta perspectiva, pacientes, familiares e equipes de profissionais têm sido, cada vez mais, enfatizados como parte integrante e necessária na avaliação de serviços. No entanto, pacientes e suas famílias têm recebido atenção privilegiada, em detrimento do estudo sobre as repercussões sentidas pela equipe de profissionais.

Borges et al..$^{10}$ alertam às organizações sobre os custos emocionais e as necessidades que envolvem a saúde mental dos trabalhadores da área da saúde. Salientam que, para uma instituição atingir seus objetivos de excelência no atendimento e qualidade nos serviços prestados, é necessário ter profissionais satisfeitos e que gozem de boa qualidade de vida.

Uma questão extremamente importante em relação ao impacto da carga de trabalho diz respeito ao prejuízo que o estresse ocupacional e o sofrimento psíquico podem desempenhar na eficácia dos tratamentos fornecidos aos pacientes. Estes fatores são considerados custos "não-econômicos" na avaliação de programas e tratamentos?.

Embora profissionais da área da saúde mental compartilhem várias atividades, cada profissão tem suas peculiaridades, tanto na formação quanto no exercício profissional. Dessa forma, além das vicissitudes inerentes à tarefa específica de cada profissão, as fases e as etapas em que o profissional se encontra podem produzir desgastes específicos ${ }^{3}$.

Partindo desta perspectiva, este estudo se propõe a identificar a relação entre carga de trabalho em saúde mental, satisfação profissional, qualidade de vida e presença de sintomas psiquiátricos menores. Para isso, a hipótese em avaliação considera que o impacto da sobrecarga de trabalho é aspecto preditivo de menor qualidade de vida e menor satisfação profissional, mesmo quando controlado para a presença de sintomatologia psiquiátrica.

\section{MÉTODO}

Estudo transversal com 203 profissionais de saúde mental do Departamento de Psiquiatria da Universidade Federal de São Paulo/Escola Paulista de Medicina, realizado em 2006.

Fizeram parte da amostra os seguintes profissionais: médicos, psicólogos, assistentes sociais, enfermeiros, auxiliares de enfermagem, terapeutas ocupacionais, fonoaudiólogo, antropólogo, dentista e educadores. Foram incluídos profissionais de saúde mental com atuação direta com os pacientes psiquiátricos.

Foram distribuídos 220 questionários, havendo retorno de 206 (93,6\%). Dez questionários retornaram em branco, por questões pessoais, e quatro foram excluídos por estarem incompletos.

Não houve diferença estatisticamente significativa ( $p>0,05)$ entre os 203 sujeitos incluídos no estudo e os 14 excluídos, no que diz respeito ao tipo de serviço e à categoria profissional.

Foram utilizados os seguintes instrumentos.

\section{Questionário sociodemográfico ocupacional}

Questionário com os seguintes dados: idade, sexo, raça, estado civil, escolaridade, ocupação, renda familiar, profissão, tempo de experiência profissional, local de trabalho, rendimentos, grau hierárquico e tipo de clientela. 


\section{IMPACTO-BR E SATIS-BR}

São instrumentos auto-aplicáveis que foram traduzidos e adaptados para a cultura brasileira pelo Laboratório de Investigações em Saúde Mental da Universidade de São Paulo e a análise e a validação das escalas foram feitas no Laboratório de Pesquisa em Saúde Mental da Universidade de São João Del Rei (LAPSAM), sendo partes de um projeto multicêntrico da OMS 8,9 .

O IMPACTO-BR contém 34 questões que visam a avaliar a sobrecarga sentida pelos profissionais de saúde mental, em conseqüência do trabalho diário com pessoas portadoras de transtornos psiquiátricos. Entre os itens, incluem-se as questões quantitativas da escala propriamente dita que avaliam o grau de sobrecarga sentida pela equipe, as questões descritivas que colhem informações complementares sobre as repercussões deste tipo de trabalho em suas vidas e as questões sociodemográficas. Cada um dos itens contém cinco níveis de respostas dispostas em escala ordinal tipo Likert de 5 pontos. O IMPACTO-BR apresenta alfa de Cronbach de 0,879.

O SATIS-BR contém 69 questões, sendo elas: 1) questões quantitativas, que visam a avaliar o grau de satisfação da equipe em relação aos serviços oferecidos e às condições de trabalho na instituição em que atuam; 2) questões qualitativas e descritivas que complementam estas informações e que se referem à opinião da equipe sobre os serviços; e 3) questões que avaliam os dados sociodemográficos. A escala global apresenta alfa de Cronbach de 0,899.

\section{Short form health survey (SF-36)}

É um instrumento genérico de avaliação de qualidade de vida multidimensional, já traduzido e validado para a população brasileira, formado por 36 itens englobados em oito domínios, sendo quatro referentes às áreas de saúde física (capacidade funcional, aspectos físicos, dor, estado geral de saúde) e quatro relativos às áreas de saúde mental (vitalidade, aspectos sociais, aspectos emocionais e saúde mental). Para cada questão é atribuído um valor em notas de oito domínios que variam de 0 (zero) a 100 (cem), em que $0=$ pior e $100=$ melhor qualidade de vida para cada domínio ${ }^{11,12}$. Este instrumento foi validado no Brasil por Cicconelli13.

\section{Self report questionaire-20}

Foi desenvolvido pela OMS como instrumento de rastreamento de sintomas psiquiátricos menores, e validado no Brasil por Mari e Willians ${ }^{14}$. Consiste de 20 itens auto-aplicáveis com escore zero ou 1 relacionados aos sintomas neuróticos, em que o escore zero indica que o sintoma esteve ausente nos últimos 30 dias e o escore 1, que esteve presente. Um escore total é obtido pela soma dos pontos. A validação do instrumento para a realidade brasileira apresentou sensibilidade de 0,83 e especificidade de 0,80, com ponto de corte $7 / 8$, ou seja, todo escore maior que 7 é considerado caso, o que indica a presença de sintomas psiquiátricos menores.

O projeto foi aprovado pelo Comitê de Ética da Universidade Federal de São Paulo. Foi realizado contato inicial com todos os serviços do departamento de psiquiatria, neste contato inicial, a pesquisadora solicitou autorização para a realização da pesquisa com as equipes de profissionais. Em cada serviço foi realizada reunião para explicar os objetivos do estudo. Individualmente, cada profissional respondeu aos instrumentos na presença do entrevistador, em horário agendado. Aos participantes foi garantido sigilo e anonimato, bem como esclarecidas todas as informações que julgaram necessárias a respeito da presente pesquisa. Foi solicitada a anuência dos participantes por meio da assinatura do Termo de Consentimento Livre e Esclarecido.

Foi realizada a distribuição de freqüências das respostas e o cálculo de tendência central da amostra total. O impacto da sobrecarga de trabalho foi avaliado em função das variáveis sociodemográficas e ocupacionais, com comparação das medianas utilizando o teste T para amostras independentes ou ANOVA. Foram, então, desenvolvidos estudos de correlação da sobrecarga de trabalho em função dos domínios de qualidade de vida, da presença de transtornos psiquiátricos menores e do nível de satisfação profissional, utilizando o teste de correlação de Spearman.

Para se avaliar se o impacto da sobrecarga de trabalho com paciente psiquiátrico é fator preditivo de pior qualidade de vida, maior sintomatologia psiquiátrica e menor satisfação profissional, conduz-se estudos de regressão linear múltipla, uma vez que a presença de sintomatologia psiquiátrica também afeta a qualidade de vida e a satisfação profissional. Em um segundo momento, o escore de sintomatologia psiquiátrica foi incluído no modelo de regressão como mediador da relação entre impacto e qualidade de vida, e impacto e satisfação profissional.

\section{RESULTADOS}

Participaram do estudo 203 profissionais, sendo: 46 psiquiatras, 111 psicólogos, 19 terapeutas ocupacionais, oito assistentes sociais, quatro enfermeiros, três auxiliares de enfermagem, uma fonoaudióloga, um dentista, um antropólogo e nove educadores. Os profissionais eram predominantemente do sexo feminino $(75,4 \%)$, com idade média de 33,7 anos ( $\mathrm{DP}=9$, variação de 21 a 59 anos); $86,7 \%$ da raça branca; $50 \%$ solteiros; $71,4 \%$ cursando ou com pós-graduação concluída (Tabela 1); e 15,8\% apresentaram sintomas de transtorno psiquiátrico menor. 
Tabela 1. Dados sociodemográficos dos profissionais.

\begin{tabular}{|c|c|c|}
\hline & $\mathrm{n}$ & $\%$ \\
\hline \multicolumn{3}{|l|}{ Sexo } \\
\hline Masculino & 50 & 24,6 \\
\hline Feminino & 153 & 75,4 \\
\hline \multicolumn{3}{|l|}{ Idade } \\
\hline Média \pm DP & - & $33,7 \pm 9,0$ \\
\hline Mediana & - & 31 \\
\hline \multicolumn{3}{|l|}{ Raça } \\
\hline Branca & 176 & 86,7 \\
\hline Outra & 27 & 13,3 \\
\hline \multicolumn{3}{|l|}{ Estado civil } \\
\hline Casado/amasiado & 86 & 42,4 \\
\hline Solteiro, divorciado, viúvo & 117 & 57,6 \\
\hline \multicolumn{3}{|l|}{ Grau de instrução } \\
\hline Até ensino superior completo & 58 & 28,6 \\
\hline Pós-graduação completa & 70 & 34,5 \\
\hline Pós-graduação incompleta & 75 & 36,9 \\
\hline \multicolumn{3}{|l|}{ Tipo de vínculo de trabalho } \\
\hline Assalariado & 66 & 32,5 \\
\hline Autônomo & 20 & 9,9 \\
\hline Estagiário & 30 & 14,8 \\
\hline Voluntário & 69 & 34,0 \\
\hline Outros & 18 & 8,9 \\
\hline Total & 203 & 100,0 \\
\hline
\end{tabular}

Verificou-se que o escore médio de satisfação global foi 3,59 $\pm 0,48$, o que resulta satisfação média. Em relação às subescalas, os fatores com maior pontuação foram satisfação da equipe com a qualidade dos serviços oferecidos com 3,89 $\pm 0,53$ e satisfação com a equipe e relacionamento no serviço com escore 3,82 $\pm 0,70$. Já o fator que gerou menor satisfação está relacionado às condições de trabalho com 3,25 \pm 0,59 (Tabela 2).

Tabela 2. Escores médios de impacto no trabalho e satisfação profissional.

\begin{tabular}{lccc}
\hline & $\begin{array}{c}\text { Variação } \\
\text { da medida }\end{array}$ & Média & (DP) \\
\hline Impacto global & $1-4$ & 1,85 & 0,536 \\
Impacto físico e mental na equipe & $1-4$ & 1,68 & 0,655 \\
$\quad$ Impacto sobre o funcionamento da equipe & $1-4$ & 1,84 & 0,591 \\
Impacto emocional & $1-4$ & 2,23 & 0,644 \\
Satisfação global & $2-5$ & 3,59 & 0,485 \\
Satisfação da equipe com qualidade serviços oferecidos & $2-5$ & 3,89 & 0,532 \\
Satisfação da equipe com a participação no serviço & $1-5$ & 3,50 & 0,684 \\
Satisfação da equipe e condições de trabalho & $2-5$ & 3,25 & 0,590 \\
Satisfação da equipe e relacionamento no serviço & $1-5$ & 3,82 & 0,701 \\
\hline
\end{tabular}

Em relação ao impacto do trabalho, o estudo obteve escore global de 1,85 \pm 0,54, resultando baixo impacto do trabalho. Quanto às subescalas, o fator relacionado ao impacto emocional causado pelo trabalho obteve a maior pontuação, com média de 2,23 \pm 0,64 (Tabela 2).

O valor do coeficiente de correlação de Pearson ( $r=$ -0,422) sugere que há associação negativa entre a satisfação profissional e o impacto do trabalho, ou seja, quando se aumenta o impacto o nível de satisfação diminui $(p<0,05)$.

Ainda sobre os níveis de satisfação profissional, outro dado observado foi a associação entre tempo de serviço e satisfação com o relacionamento no serviço. Profissionais que têm entre cinco e dez anos e mais de dez anos de trabalho no mesmo departamento apresentam níveis menores de satisfação com o relacionamento mantido no local de trabalho, e profissionais que trabalham mais de 20 horas semanais não se sentem tão satisfeitos com a qualidade dos serviços oferecidos, além de apresentarem escores mais rebaixados nos subitens: dor (42,3\%), estado geral de saúde $(63,3 \%)$ e vitalidade $(58,4 \%)$ da escala SF36, do que os profissionais que trabalham menos de 20 horas semanais.

Entre as profissões pesquisadas, foi observado que o impacto do trabalho é mais sentido pelos terapeutas ocupacionais e pelos assistentes sociais. Estes profissionais também apresentam escores mais elevados na subescala referente ao impacto emocional, porquanto $31,6 \%$ dos terapeutas ocupacionais e $37,5 \%$ dos assistentes sociais apresentam transtornos psiquiátricos menores, diferentemente das outras profissões em que a média foi de 15,8\% (Tabela 3).

Os resultados obtidos com o SRQ-20 demonstram que a predominância de transtorno psiquiátrico menor é maior no sexo feminino (17,6\%), com idade média de 31,8 anos, em autônomos (35\%) e estagiários (23,3\%) e nos profissionais que trabalham entre 20 e 30 horas semanais (25\%).

Em relação à idade, foram encontradas diferenças estatisticamente significantes. Quanto maior a idade menor o impacto emocional e menor o impacto sobre o funcionamento da equipe $(p<0,05)$.

Tabela 3. Medidas descritivas dos escores de comorbidades psiquiátricas por profissão.

\begin{tabular}{|c|c|c|c|c|c|c|c|c|c|c|}
\hline & \multirow{2}{*}{$\mathrm{n}$} & \multirow{2}{*}{ Média } & \multirow{2}{*}{ DP } & \multirow{2}{*}{ Mediana } & \multirow{2}{*}{ Mínimo } & \multirow{2}{*}{ Máximo } & \multicolumn{2}{|c|}{ Negativo } & \multicolumn{2}{|c|}{ Positivo } \\
\hline & & & & & & & $\mathrm{n}$ & $\%$ & $\mathrm{n}$ & $\%$ \\
\hline Médico & 42 & 1,2 & 0,4 & 1,0 & 1,0 & 2,0 & 35 & 83,3 & 7 & 16,7 \\
\hline Psicólogo & 111 & 1,1 & 0,4 & 1,0 & 1,0 & 2,0 & 95 & 85,6 & 16 & 14,4 \\
\hline Terapeuta ocupacional & 19 & 1,3 & 0,5 & 1,0 & 1,0 & 2,0 & 13 & 68,4 & 6 & 14,4 \\
\hline Assistente social & 8 & 1,4 & 0,5 & 1,0 & 1,0 & 2,0 & 5 & 62,5 & 3 & 37,5 \\
\hline Auxiliar de enfermagem & 3 & 1,0 & 0,0 & 1,0 & 1,0 & 1,0 & 3 & 100,0 & 0 & 0,0 \\
\hline Enfermeiro & 4 & 1,0 & 0,0 & 1,0 & 1,0 & 1,0 & 4 & 100,0 & 0 & 0,0 \\
\hline Outros & 16 & 1,0 & 0,0 & 1,0 & 1,0 & 1,0 & 16 & 100,0 & 0 & 0,0 \\
\hline Total & 203 & 1,2 & 0,4 & 1,0 & 1,0 & 2,0 & 171 & 84,2 & 32 & 15,8 \\
\hline
\end{tabular}

$p$ (teste generalizado de Fisher) $=0,040$ 


\section{DISCUSSÃO}

A partir das informações apresentadas pode-se compreender os índices alcançados nas escalas SATIS-BR e IMPACTO-BR de média satisfação profissional e baixo impacto do trabalho pelo fato de a pesquisa ter sido realizada em uma universidade federal, onde existe, por parte dos profissionais, sentimento forte de orgulho por atuar na instituição. Os índices refletem a opinião dos participantes que relatam orgulho e confiança em trabalhar com profissionais competentes e referenciados na área, por presenciarem bons resultados nos tratamentos empregados e por terem a oportunidade de aprender e participar de estudos e pesquisas constantemente, se mantendo sempre atualizados.

Por outro lado, os resultados obtidos com as respostas às perguntas qualitativas, que se intercalavam no decorrer da atual pesquisa, demonstram que o nível de satisfação profissional poderia ser maior se houvessem mais investimentos para a melhora da infra-estrutura física dos serviços em geral, ter um local mais apropriado, limpo e agradável para atendimento dos pacientes, ou seja, quantidade de salas e tamanhos adequados à demanda de pacientes.

Outro fator negativo está relacionado ao número de profissionais contratados que é insuficiente para a demanda dos serviços e a baixa remuneração.

Estes resultados estão em concordância com recentes estudos brasileiros que ao pesquisarem outras instituições de saúde mental encontraram escores intermediário de satisfação e baixo impacto ${ }^{6,8,15}$.

Lancman et al. ${ }^{16}$, em estudo sobre a ergonomia e a psicodinâmica do trabalho, enfatizam que a discussão sobre a compreensão do trabalho continua a ser um desafio para os diversos pesquisadores, que, até então, acessam apenas as conseqüências nas mentes e nos corpos dos trabalhadores e desconhecem aspectos importantes sobre o ato de trabalhar.

Embora profissionais da área da saúde mental compartiIhem várias atividades, cada profissão tem suas peculiaridades tanto na formação quanto no exercício profissional. Dessa forma, além das vicissitudes inerentes à tarefa específica de cada profissão, as fases e etapas em que o profissional se encontra na carreira pode produzir desgastes específicos ${ }^{3}$.

Em relação aos resultados obtidos com esta pesquisa, destacam-se os dados referentes aos terapeutas ocupacionais e assistentes sociais que mostraram sentir, de maneira mais exacerbada, o impacto da carga de trabalho em relação aos outros profissionais pesquisados, além de apresentarem índices mais elevados de transtornos psiquiátricos menores do que os demais profissionais. Isso pode estar relacionado às peculiaridades do trabalho e a escolha de atuação com a área da saúde mental. O trabalho dos terapeutas ocupacionais tem como objetivo a reabilitação do indivíduo, contribuindo na avaliação, no planejamento e no tratamento de diversas disfunções físicas, mentais, sociais e de desenvolvimento. Algumas hipóteses podem esclarecer os resultados obtidos: falta de reconhecimento profissional pelos demais profissionais da equipe, falsa concepção da equipe de que a terapia ocupacional é apenas distração e recreação para os pacientes incapacitados e ambigüidade em relação ao papel profissional, muitas vezes associado a de fisioterapeutas, e outras, ao de psicoterapeutas.

Sweeney e Nichols ${ }^{17}$, em estudo sobre o estresse em terapeutas ocupacionais, verificaram que os profissionais que optaram em trabalhar com saúde mental apresentaram altos índices de estresse. Os fatores que contribuíram para os elevados índices foram constante exposição às angústias e dificuldades dos pacientes, freqüente exposição àqueles que têm má qualidade de vida e resistência que muitos pacientes têm em mudar condições de vida que antes não traziam conseqüências desagradáveis, mas que agora contribuem para situações difíceis. Geralmente, as intervenções em terapia ocupacional requerem ativa cooperação e engajamento do paciente por longo período e contato e, principalmente na área da saúde mental, requerem substancial autonomia e criatividade.

Em relação às assistentes sociais, a literatura evidencia os riscos ocupacionais relacionados aos elevados índices de estresse derivados dos efeitos do ambiente de trabalho e características da profissão, em que há paradoxo entre vivenciar situações de poder em relação ao cliente, e por outro lado, falta de autonomia em relação à instituição e aos recursos ${ }^{18}$.

Wood (1982), citado por Pottage e Huxley ${ }^{19}$, acredita que as pessoas que optam por esta profissão possuem certas características que são pilares para o serviço social, como acreditar na justiça social, no bem-estar das pessoas, no não julgamento das atitudes, no respeito pelas escolhas e autonomia, no senso de responsabilidade pelos outros e no profundo concernimento pelo sofrimento humano. Outro estudo sugere que pessoas com estas características acabam se envolvendo profundamente com o outro e, muitas vezes, estão predispostos à exaustão emocional ${ }^{20}$.

Corroborando os resultados do estudo sobre estes dois grupos de profissionais, Lloyd e King ${ }^{21}$ pesquisaram o burnout por meio do Maslach Burnout Inventory com um grupo de 304 profissionais de terapia ocupacional e serviço social. Os dois grupos mostraram altos índices de exaustão emocional, moderada despersonalização e baixa realização pessoal.

Os subitens dor, estado geral de saúde e vitalidade, avaliado abaixo da média no quesito qualidade de vida, alertam para conseqüência muitas vezes não associada ao trabalho, mas que podem estar relacionadas à ergonomia e à psicodinâmica do trabalho, conforme estudos realizados por Lancman ${ }^{16,22}$.

Como esperado e observado em outros estudos ${ }^{6,23}$, quanto maior a idade, menor o impacto emocional no trabalho, o que sugere que a experiência aumenta a segurança nas decisões tomadas e maior controle sobre a demanda de trabalho, diminuindo o estresse e a exaustão emocional. 
Os resultados obtidos no SRQ-20 estão abaixo dos valores apresentados em outros estudos nacionais ${ }^{14,24}$. No entanto, a prevalência em mulheres, acima dos 30 anos se compara aos resultados anteriormente estudados.

O estudo apresenta algumas limitações, apesar de a amostra geral ser de 203 profissionais, não eram idênticos os números de categorias de profissionais, dificultando maiores comparações entre as diferentes profissões.

Os resultados não podem ser generalizados para outras instituições, visto o estudo ter sido realizado em uma única instituição e possuir especificidades que não permitem generalizações com profissionais que atuem em outras instituições, sejam elas públicas ou privadas.

Por ser pesquisa quantitativa de corte transversal, com questionários auto-aplicáveis, fica-se sujeito à confiabilidade das informações prestadas, assim como ao estado emocional do participante no momento da pesquisa.

\section{CONCLUSÃO}

Os dados obtidos com esta pesquisa demonstram que os profissionais que atuam na área da saúde mental sentem-se mais satisfeitos em relação à qualidade dos serviços oferecidos e ao trabalho realizado em equipe. No entanto, os resultados alertam para a condição do trabalho, isso se traduz na necessidade de mais investimentos na infra-estrutura física dos serviços, assim como a adequação do número de recursos humanos e materiais para atender, de maneira satisfatória, a demanda de pacientes.

Para melhor identificação dos resultados aqui pesquisados, considera-se interessante estudo comparativo entre satisfação dos pacientes com os serviços oferecidos versus impacto do trabalho e satisfação dos profissionais do mesmo serviço, no qual teria-se a oportunidade de identificar, de maneira mais focada e objetiva, fatores que contribuam para a qualidade do atendimento e aspectos que precisem de mais atenção.

\section{AGRADECIMENTOS}

À Fundação de Amparo a Pesquisa do Estado de São Paulo (Fapesp) que financiou esta pesquisa sob o processo $n^{\circ}$ 05/57.405-6. A todos os voluntários que aceitaram participar do estudo. Ao Departamento de Psiquiatria da Universidade Federal de São Paulo, por permitir a realização da pesquisa em todos os seus ambulatórios.

\section{REFERÊNCIAS}

1. Brasil. Ministério da Saúde. Secretaria de Atenção à Saúde. DAPE. Coordenação Geral de Saúde Mental. Reforma psiquiátrica e política de saúde mental no Brasil. Documento apresentado à Conferência Regional de Reforma dos Serviços de Saúde Mental: 15 anos depois de Caracas. OPAS. Brasília, 2005 [acesso em 2008 Mar 28]. Disponível em: http://portal. saude.gov.br/portal/arquivos/pdf/Relatorio15\%20anos\%20Caracas.pdf.

2. Cordeiro H. Descentralização, universalidade e equidade nas reformas da saúde. Cien Saude Colet. 2001;6(2):319-28.

3. Nogueira-Martins LA. Saúde mental dos profissionais de saúde. In: Botega NJ (Org.). Prática psiquiátrica no hospital geral: interconsulta e emergência. Porto Alegre: Artmed; 2002. p. 130-44.

4. Abreu KL, Stoll I, Ramos LS, Baumgardt RA, Kristensen CH. Estresse ocupacional e síndrome de Burnout no exercício profissional da psicologia. Psic C Prof. 2002;22:23-30.

5. Benevides-Pereira AMT. Burnout: quando 0 trabalho ameaça o bem-estar do trabalhador. São Paulo: Casa do Psicólogo; 2002.

6. Rebouças D, Legay LF, Abelha L. Satisfação com o trabalho e impacto causado nos profissionais de serviços de saúde mental. Rev Saude Publica. 2007;41(2):244-50.

7. World Health Organization (WHO). The World Health Report 2001. Mental Health: New Understanding, New Hope [acesso em 2008 Mar 25]. Disponível em: http://www.who.int/ whr/2001/en/

8. Pitta AM. Qualidade de serviços de saúde mental: desafios para a epidemiologia. J Bras Psiquiatr. 1996;45(6):313-21.

9. Bandeira M, Pitta AMF e Mercier C. Escalas brasileiras de avaliação da satisfação (SATIS-BR) e da sobrecarga (IMPACTO-BR) da equipe técnica em serviços de saúde mental. J Bras Psiq. 2000;49(4):105-15

10. Borges LO, Argolo JCT, Pereira ALS, Machado EAP, Silva WSA. Síndrome de Burnout e os valores organizacionais: um estudo comparativo em hospitais universitários. Psicol Reflex Crit. 2002;45(1):189-200

11. Hays RD, Wells KB, Sherbourne CD, Rogers W, Spritzer K. Functioning and well-being outcomes of patients with depression compared with chronic general medical illnesses. Arch Gen Psychiatry. 1995;52:11-9.

12. Ware JJ, Sherbourne CD. The MOS 36-item short-form health survey (SF-36). I. Conceptual framework and item selection. Med Care. 1992;30:473-83.

13. Cicconelli RM. Tradução e validação para o português do Medical Outcomes Study 36-Item Short-Form Health Survey (SF-36) [tese]. São Paulo: Universidade Federal de São Paulo. Escola Paulista de Medicina, 1997.

14. Mari JJ, Williams P. A validity study of a psychiatric screening questionnaire (SRQ-20) in primary care in the city of São Paulo. Br J Psychiatry. 1986;148:23-6.

15. Ishara S. Equipes de saúde mental: avaliação da satisfação e do impacto do trabalho em hospitalização integral e parcial. [tese]. Ribeirão Preto: Universidade de São Paulo, 2007.

16. Lancman S, Sznelwar L, Santos MC, Alvarinho E, Wu MJ. Análise do trabalho e do serviço de limpeza hospitalar: contribuiçōes da ergonomia e da psicodinâmica do trabalho. Rev Produção. 2004;14(3):45-57.

17. Sweeney G, Nichols K. Stress experiences of occupational therapists in mental health practice arenas: a review of the literature. Int I Soc Psychiatry. 1996;42:132-40,

18. Jones F, Fletcher B, Ibbetson K. Stresses and strains amongst social workers, demands, supports, constraints and psychological health. Br J Soc Work. 1991;21(5):443-70

19. Pottage D, Huxley P. Stress and mental healthy social work a developmental perpective. Int J Soc Psyquiatry. 1996;42:124-31.

20. Williams CA. Empathy and burnout in male \& female helping professionals. Res Nurs Health. 1989:12(3):169-78

21. Lloyd C, King R. A survey of Burnout among australian mental health occupational therapists and social workers. Soc Psychiatry Psychiatr Epidemiol. 2004;39(9):752-7.

22. Lancman S, Jardim TA. 0 impacto da organização do trabalho na saúde mental: um estudo em psicodinâmica do trabalho. Rev Ter Ocup Univ. 2004;15(2):42-89.

23. Fothergill A, Edwards D, Burnard P. Stress, Burnout, coping and stress management in psychiatrists: findings from a systematic review. Int I Soc Psychiatry. 2004;50(1):54-65.

24. Almeida-Filho N, Mari JJ, Coutinho ESF, França J, Fernandes J, Andreoli SB, et al. Brazilian multicentric study of psychiatric morbidity: metodological features and prevalence estimates. Br J Psychiatry. 1997;171:524-9.

25. Bandeira M, Soares NL. Desinstitucionalização: estão os profissionais de saúde mental bem informados? J Bras Psiq. 1996;45(3):159-65.

26. Carvalho M, Vieira AA. Medical errors in hospitalized patients. J Pediatr. 2002;78(4):261-8.

27. Lipp MN (Org.). Pesquisas sobre stress no Brasil: saúde, ocupações e grupos de risco. São Paulo: Papirus; 1996. 